\title{
Interference effects between direct and sequential processes in the $\left({ }^{18} \mathrm{O},{ }^{16} \mathrm{O}\right)$ reaction
}

\author{
M. Cavallaro ${ }^{1 \mathrm{a}}$, F. Cappuzzello ${ }^{1,2}$, M. Bondì ${ }^{1,2}$, D. Carbone ${ }^{1,2}$, V. N. Garcia ${ }^{3}$, A.Gargano ${ }^{4}$, S.M.Lenzi ${ }^{5}$, \\ J. Lubian $^{3}$, C. Agodi ${ }^{1}$, F. Azaiez ${ }^{6}$, M. De Napoli ${ }^{7}$, A.Foti ${ }^{2,7}$, S. Franchoo ${ }^{6}$, R. Linares ${ }^{3}$, D. Nicolosi ${ }^{1,2}$, \\ M. Niikura ${ }^{6}$, J. A. Scarpaci ${ }^{6}$, S. Tropea ${ }^{1,2}$ \\ ${ }^{1}$ INFN - Laboratori Nazionali del Sud, Italy \\ ${ }^{2}$ Dipartimento di Fisica e Astronomia, Università degli Studi di Catania, Italy \\ ${ }^{3}$ Instituto de Física, Universidade Federal Fluminense, Niteroi, RJ, Brazil \\ ${ }^{4}$ INFN - Sezione di Napoli, Italy \\ ${ }^{5}$ INFN - Sezione di Padova, Italy \\ ${ }^{6}$ Institut de Physique Nucléaire, Université Paris-Sud-11-CNRS/IN2P3, Orsay, France \\ ${ }^{7}$ INFN - Sezione di Catania, Italy
}

\begin{abstract}
The ${ }^{12} \mathrm{C}\left({ }^{18} \mathrm{O},{ }^{16} \mathrm{O}\right){ }^{14} \mathrm{C}$ reaction at $84 \mathrm{MeV}$ has been studied at INFN-LNS laboratory using the MAGNEX magnetic spectrometer. High resolution energy spectra and cross-section angular distributions have been measured. DWBA calculations of both direct and sequential transfer to the ${ }^{14} \mathrm{C}$ ground state show the important role of the interference between the two processes to describe the experimental data.
\end{abstract}

\section{Introduction}

Transfer reactions have been widely used for the exploration of the nuclear structure, thanks to their strong selectivity in selecting specific degrees of freedom of the nuclear system [1-3]. One can mention for example the well established connection between one-nucleon transfer cross sections and single-particle configurations of the nuclear states. In analogy, a relation between two-nucleon transfer probabilities and pairing correlations in nuclei is also expected.

However the extraction of structure information from two-nucleon transfer cross-sections is not straightforward, especially when dealing with heavy-ions. This is due both to experimental difficulties to measure excitation energy spectra at high resolution and in a large angular range, in order to extract angular distributions, and also to the complex interpretation of the data. For example the use of optical potential extracted from fits of elastic scattering data often fails in describing transfer cross-sections. Moreover one should take into account the coupling with inelastic excitations (coupled-channels corrections) and the contribution of a multistep route involving two successive single-neutron transfer, which introduce couplings with intermediate partitions.

As a consequence, the analyses present in literature required the use of arbitrary scaling factor, often called "unhappiness factor", much larger that one and even as high as hundreds to reproduce the experimental angular distributions $[4,5]$.

\footnotetext{
${ }^{\mathrm{a}}$ Corresponding author: manuela.cavallaro@lns.infn.it
} 
In the present paper, a DWBA analysis of the angular distributions is performed in the special case of the ${ }^{12} \mathrm{C}\left({ }^{18} \mathrm{O},{ }^{16} \mathrm{O}\right){ }^{14} \mathrm{C}_{\text {g.s. }}$ reaction at $84 \mathrm{MeV}$ incident energy. The use of the São Paulo double folding potential in an extreme cluster model, where the two transferred neutrons are coupled anti-parallel and the relative motion is frozen allows to reproduce in a reasonable way the observed cross-section and the oscillatory pattern of the angular distributions. Taking into account the interference effects between the direct and sequential mechanisms, the agreement improves, allowing for the first time the description of heavy-ion transfer cross-section without the need of any arbitrary scaling factor.

\section{The Experiment}

The experiment was carried out at the INFN-LNS laboratory in Catania. A beam of ${ }^{18} \mathrm{O}^{6+}$ ions was accelerated at $84 \mathrm{MeV}$ incident energy by the Tandem Van de Graaff.

A $99 \%$ enriched $49 \mu \mathrm{g} / \mathrm{cm}^{2}$ self supporting target of ${ }^{12} \mathrm{C}$ was used. The ${ }^{16} \mathrm{O}$ ejectiles produced in the collisions were momentum analyzed by the MAGNEX spectrometer [6], working in full acceptance mode $(\Omega \sim 50 \mathrm{msr}$ solid angle and $\Delta \mathrm{p} / \mathrm{p} \sim 24 \%$ momentum acceptance). The experiment was performed at four angular setting, with the spectrometer optical axis centered at $6^{\circ}, 12^{\circ}, 18^{\circ}$ and $24^{\circ}$, covering each time an aperture of about $12^{\circ}$, thus guaranteeing about $6^{\circ}$ angular overlaps. The magnetic fields were set in order to accept the Oxygen ions with charge state between $5^{+}$and $8^{+}$. The lower charge states correspond to less energetic particles transmitted through the accepted magnetic rigidity interval.

The reaction ejectiles were detected and identified by the focal plane detector (FPD) $[7,8]$ through the simultaneous measurement of their position and angle, the energy loss in the gas section and the residual energy on the wall of 54 silicon pad detectors mounted at the end of the FPD. The data reduction technique [9] and the performances of the whole system are described in Refs. [10-12].

The absolute cross section angular distribution for the transition ${ }^{12} \mathrm{C}\left({ }^{18} \mathrm{O},{ }^{16} \mathrm{O}\right){ }^{14} \mathrm{C}_{\text {g.s. }}$ is shown in Figure 1. It exhibits a pronounced oscillating pattern, characteristic of the well known $L=0$ angular momentum transfer.

\section{DWBA analysis of the angular distribution}

We performed Exact Finite Range (EFR) Distorted Wave Born Approximation (DWBA) cross-section calculations of the ${ }^{18} \mathrm{O}+{ }^{12} \mathrm{C} \rightarrow{ }^{16} \mathrm{O}+{ }^{14} \mathrm{C}$ transitions using the FRESCO code [13].

Both the direct transfer of a cluster of two neutrons coupled with spin $S=0$ and the sequential transfer of the two neutrons, introducing the intermediate partition ${ }^{13} \mathrm{C}+{ }^{17} \mathrm{O}$, were calculated.

The São Paulo double folding potential (SPP) was used as real part in the optical model [14]. The matter diffuseness for the ${ }^{18} \mathrm{O}$ and ${ }^{17} \mathrm{O}$ nuclei was taken by Refs. [15,16]. The imaginary part of the optical potential was built with the SPP shape. The wave functions used in the form factor calculations were generated by a Woods-Saxon potential, whose depth was adjusted to fit the experimental oneand two-neutron separation energies.

Shell model calculations were also performed in order to provide the spectroscopic amplitudes in the $1 \mathrm{p}_{1 / 2}, 1 \mathrm{~d}_{5 / 2}$ and $2 \mathrm{~s}_{1 / 2}$ model space. In particular, a spectroscopic factor of 0.83 is predicted for the ${ }^{12} \mathrm{C}_{\text {g.s. }} \rightarrow{ }^{14} \mathrm{C}_{\text {g.s. }}$ transition with the two neutrons in $\left(\mathrm{p}_{1 / 2}\right)^{2}$ configuration. This value is well in agreement to what found in literature.

The results of the DWBA calculations, both direct and sequential, are shown in Figure 1 superimposed to the experimental data in the case of the transition to the ${ }^{14} \mathrm{C}_{\mathrm{g} . s .}$. 


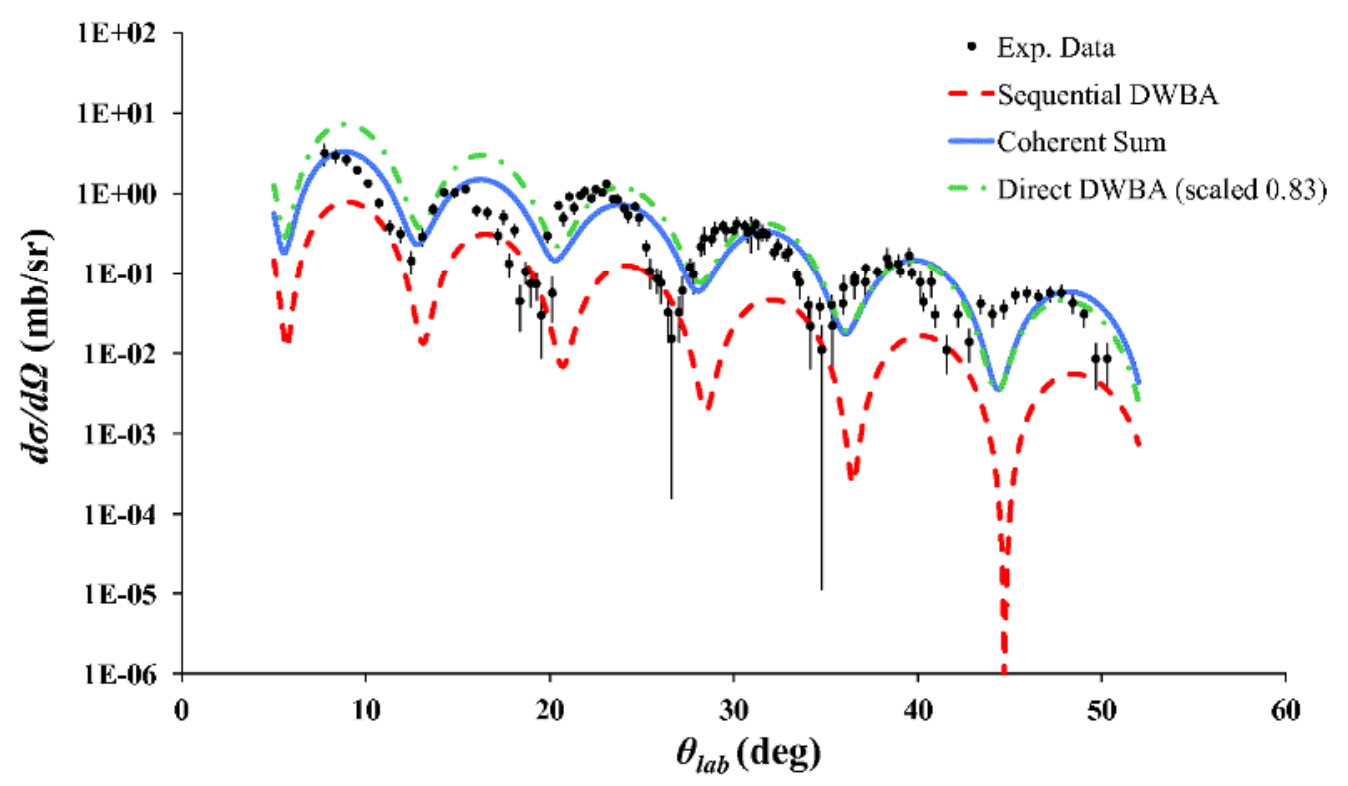

Figure 1. (Color online) Cross-section angular distribution for the transition ${ }^{12} \mathrm{C}\left({ }^{18} \mathrm{O},{ }^{16} \mathrm{O}\right){ }^{14} \mathrm{C}_{\text {g.s. }}$ at $84 \mathrm{MeV}$. The experimental data (black dots) are compared to the results of direct DWBA calculations (green dot-dashed curve), sequential DWBA (red dashed) and to the coherent sum of the two contributions with a phase shift linearly dependent on the scattering angle.

The direct DWBA calculation of Fig. 1, made taking into account the spectroscopic factors, gives a quite good description of the order of magnitude of the experimental cross-section, without the need of any arbitrary scaling factor. The sequential transfer, instead, underestimates the measured crosssection even of one order of magnitude at large angles, while it appears stronger at small angles.

The present analysis shows that in the ${ }^{12} \mathrm{C}\left({ }^{18} \mathrm{O},{ }^{16} \mathrm{O}\right){ }^{14} \mathrm{Cg}$.s. transition, the direct transfer of a pair of neutrons is dominant compared to the successive (two-step) processes. The presence of neutronneutron correlations in the ${ }^{18} \mathrm{O}$ ground state determines a sizable amount of cluster configurations in the ground state wave function of ${ }^{14} \mathrm{C}$ populated by the $\left({ }^{18} \mathrm{O},{ }^{16} \mathrm{O}\right)$ reaction, similarly to what happens in the transfer of more tightly bound systems such as $\alpha$ particles. Other works, for instance the one of Ref. [17], have discussed the effects of the simultaneous and sequential two-neutron transfer even with different results. However one should notice that in those cases, the colliding systems (e.g. ${ }^{16} \mathrm{O}+$ ${ }^{208} \mathrm{~Pb}$ ) were two closed shell nuclei and did not have a preformed pair of neutrons in the initial wave functions. Thus the dominance of a pair transfer in those systems is not foreseen.

In the present data, the coherent sum of the two processes, accounting for an arbitrary phase shift linearly depending on the scattering angle, was also performed and is shown in Figure 1. The dependence of the phase shift $\delta$ on the angle has been extracted in order to fit the experimental data. The result is a destructive interference at small angles that slowly becomes constructive at large angles. The value of the phase shift decreases in the explored angular region from $\delta=180^{\circ}$ to $\delta=70^{\circ}$.

The present approach represents a simple way to estimate the effects to be expected from a fully coherent treatment. One should notice that, according to the perturbative formalism of two-particle transfer, the total transition amplitude up to second order should contain also the full contribution due to the non-orthogonality terms between the states of initial, intermediate and final partitions [1]. Such a complete treatment, developed for example for $(\mathrm{p}, \mathrm{t})$ reactions in Ref. [18], is missing in the present case, where different model spaces were used in the direct and sequential calculations.

In addition, the coupling to the inelastic channels, neglected in the present DWBA approach, are known to play an important role in describing heavy-ion transfer reactions [4]. The performance of complete Coupled Reaction Channel calculations is the next step of this study [19]. 


\section{References}

1. G.R. Satchler, "Direct Nuclear Reactions", Oxford University Press, 1983

2. S. Kahana and A.J. Baltz, One- and Two-Neutron Transfer Reactions with Heavy Ions, in: M. Baranger and E. Vogt (Eds.), Advances in Nuclear Physics Vol. 9, Plenum Press, New York, 1977, pp. 1-122.

3. N. Anyas-Weiss, et al., Phys. Rep. 12, no.3, 201 (1974).

4. M.-C. Lemaire and K.S. Low, Phys. Rev. C 16, 183 (1977).

5. M.C. Mermaz, et al., Phys. Rev. C 20, 2130 (1979).

6. F. Cappuzzello et al., MAGNEX: an innovative large acceptance spectrometer for nuclear reaction studies in: Magnets: Types, Uses and Safety, Nova Publisher Inc., New York, 2011, pp $1-63$.

7. M. Cavallaro, et al., Eur. Phys. J. A (2012) 48: 59.

8. D. Carbone, et al., Eur. Phys. J. A (2012) 48: 60.

9. F. Cappuzzello, et al., Nucl. Instr. and Meth. A 638, 74 (2011).

10. F. Cappuzzello, et al., Nucl. Instr. and Meth. A 621, 419 (2010).

11. M. Cavallaro, et al., Nucl. Instr. and Meth. A 637, 77 (2011).

12. M. Cavallaro, et al., Nucl. Instr. and Meth. A 648, 46 (2011).

13. J. I. Thompson, http://www.fresco.org.uk.

14. L.C. Chamon, et al., Phys. Rev. Lett. 79, 5218 (1997).

15. J. F. P. Huiza et al., Phys. Rev. C 82, 054603 (2010).

16. E. Crema et al., Phys. Rev. C 84, 024601 (2011).

17. B. F. Bayman and Jongsheng Chen, Phys. Rev. C 26, 1509 (1982).

18. G. Potel, et al., Phys. Rev. Lett. 107, 092501 (2011)

19. M. Cavallaro, et al., Phys. Rev. C accepted. 